\title{
Effect of plasma density on diffusion rates due to wave particle interactions with chorus and plasmaspheric hiss: extreme event analysis
}

\author{
A. Sicard-Piet ${ }^{1}$, D. Boscher ${ }^{1}$, R. B. Horne ${ }^{2}$, N. P. Meredith ${ }^{2}$, and V. Maget ${ }^{1}$ \\ ${ }^{1}$ ONERA - The French Aerospace Lab, 31055 Toulouse, France \\ ${ }^{2}$ British Antarctic Survey, Cambridge, UK
}

Correspondence to: A. Sicard-Piet (angelica.sicard@onera.fr)

Received: 24 January 2014 - Revised: 11 June 2014 - Accepted: 22 July 2014 - Published: 29 August 2014

\begin{abstract}
Wave particle interactions play an important role in controlling the dynamics of the radiation belts. The purpose of this study is to estimate how variations in the plasma density can affect diffusion rates resulting from interactions between chorus waves and plasmaspheric hiss with energetic particles and the resulting evolution of the energetic electron population. We perform a statistical analysis of the electron density derived from the plasma wave experiment on the CRRES satellite for two magnetic local time sectors corresponding to near midnight and near noon. We present the cumulative probability distribution of the electron plasma density for three levels of magnetic activity as measured by Kp. The largest densities are seen near $L^{*}=2.5$ while the smallest occur near $L^{*}=6$. The broadest distribution, corresponding to the greatest variability, occurs near $L^{*}=4$. We calculate diffusion coefficients for plasmaspheric hiss and whistler mode chorus for extreme values of the electron density and estimate the effects on the radiation belts using the Salammbô model. At $L^{*}=4$ and $L^{*}=6$, in the low density case, using the density from the 5 th percentile of the cumulative distribution function, electron energy diffusion by chorus waves is strongest at $2 \mathrm{MeV}$ and increases the flux by up to 3 orders of magnitude over a period of $24 \mathrm{~h}$. In contrast, in the high density case, using the density from the 95th percentile, there is little acceleration at energies above $800 \mathrm{keV}$ at $L^{*}=6$, and virtually no acceleration at $L^{*}=4$. In this case the strongest energy diffusion occurs at lower energies around $400 \mathrm{keV}$ where the flux at $L^{*}=6$ increases 3 orders of magnitude.
\end{abstract}

Keywords. Magnetospheric physics (energetic particles, trapped) - space plasma physics (charged particle motion and acceleration; wave-particle interactions)

\section{Introduction}

Wave-particle interactions play a major role in the nonadiabatic dynamics of energetic particles in the inner magnetosphere (e.g., Horne, 2002; Thorne et al., 2005; Thorne, 2010). Such interactions break the first two adiabatic invariants leading to pitch angle scattering and energy diffusion (Kennel and Petschek, 1966). They play important roles in the acceleration and loss of radiation belt electrons and contribute significantly to the large flux variations observed in the radiation belts during magnetic storms and other disturbances (e.g., Li and Temerin, 2001; Friedel et al., 2002; Thorne et al., 2013). Consequently, a better understanding of these physical processes will improve physics-based models and enable better specifications and forecasts of the radiation belts fluxes. To model wave-particle interactions, with a numerical code known as WAPI (wave-particle interaction, Boscher et al., 2007) for example, several input parameters must be properly defined including the characteristics of the waves (intensity, propagation angle) and that of the ambient plasma (electron density). The magnetic field configuration also plays an important role in wave-particle interactions (Orlova and Shprits, 2010; Ni et al., 2012; Artemyev et al., 2013), but this is not the subject of the present investigation and is not considered further here. 
Plasma waves that can interact with $\mathrm{MeV}$ electrons include whistler mode chorus, plasmaspheric hiss, magnetosonic waves and Electromagnetic Ion Cyclotron (EMIC) waves. Interaction between chorus waves and energetic electrons is an important acceleration and loss mechanism outside the plasmapause for electrons with energies from 0.1 to a few MeV (Horne et al., 2005). Interaction between plasmaspheric hiss and energetic electrons is a major loss process inside the plasmapause for electron energies from 0.1 to a few MeV (Lyons and Thorne, 1973; Meredith et al., 2007, 2009). Magnetosonic waves may also be an important acceleration mechanism (Horne et al., 2007; Meredith et al., 2008, 2009; Mourenas et al., 2013) and EMIC waves may be an important loss mechanism for $\mathrm{MeV}$ electrons (Summers and Thorne, 2003; Meredith et al., 2003; Jordanova et al., 2008). However, the roles of magnetosonic and EMIC waves in relativistic electron dynamics has yet to be fully assessed, and are not considered further here.

Whistler mode chorus is an intense electromagnetic emission observed in the frequency range from 0.1 to $0.8 \mathrm{fce}$ (electron gyrofrequency) $\left(\sim 1-10 \mathrm{kHz}\right.$ at $\left.L^{*}=4\right)$, (Tsurutani and Smith, 1977; Koons and Roeder, 1990), with a gap at 0.5 fce (Tsurutani and Smith, 1974), where fce is the equatorial electron gyrofrequency, $f_{\text {ce }}=q \boldsymbol{B} / m$, defined in terms of the electron charge, $q$, the ambient magnetic field, $\boldsymbol{B}$, and the electron mass, $m_{\mathrm{e}}$. Thus, the emissions are separated into two bands, referred to as lower band chorus $(0.1<$ fce $<0.5$ fce $)$ and upper band chorus $(0.5<f<0.8$ fce $)$. Chorus waves are largely observed outside plasmapause, where they are generated by cyclotron resonant interaction with suprathermal electrons (Li et al., 2008, 2009b) near the geomagnetic equator (Santolik et al., 2004, 2005). Chorus waves contribute to both the acceleration and loss of energetic electrons and play a significant role in radiation belt dynamics (Bortnik and Thorne, 2007). Plasmaspheric hiss is a broadband, structureless whistler mode emission that typically occurs in the frequency range from $100 \mathrm{~Hz}$ to $2 \mathrm{kHz}$ and is largely observed inside the plasmapause (Meredith et al., 2004). Resonant pitch-angle scattering of energetic electrons by plasmaspheric hiss largely accounts for the formation of the slot region that separates the inner $(1.3<L<2.5)$ and outer $(3<L<7)$ radiation belts (Lyons et al., 1972; Abel and Thorne, 1998a, b) and the quiet time decay of energetic electrons in the outer radiation belt (Meredith et al., 2006; Summers et al., 2007). Ray tracing studies suggest and observations show that the dominant source of plasmaspheric hiss is whistler mode chorus (Bortnik et al., 2008, 2011; Chen et al., 2012a; Meredith et al., 2013). The amplitude of chorus waves and plasmaspheric hiss are substorm dependent. Statistically the waves are found to be enhanced during disturbed conditions as monitored by both the Auroral Electrojet (AE) (e.g., Meredith et al., 2012) and Kp (e.g., Albert et al., 2009) indices. Radiation belt models have traditionally used wave models driven by the Kp index (e.g. Varotsou et al., 2005, 2008; Fok et al., 2007; Albert et al., 2009) and we follow this approach here.

The second main parameter to take into account in the wave-particle interaction is the electron plasma density. There are several models of the electron plasma density in the magnetosphere including the Global Plasma Core Model (GCPM) (Gallagher et al., 2000), IZMIRAN (Chasovitin et al., 1998; Gulyaeva et al., 2002), the Carpenter model (Carpenter and Anderson, 1992) and also empirical models based on CRRES data (Sheeley et al., 2001), POLAR data (Denton et al., 2004) or IMAGE Auroral Electrojet (RPI) measurements (Ozhogin et al., 2012). The electron plasma density resulting from these models can be different from one model to another and in each model the plasmapause location, the transition between the high density region within the plasmasphere and the low density region outside the plasmasphere, is different.

The purpose of this study is to estimate how variations in the plasma density can affect diffusion rates resulting from wave-energetic particle interactions and consequently the energetic electron population. In Sect. 2 we use CRRES measurements to study the distribution of electron plasma densities for selected $L^{*}$ values, magnetic local times (MLT) and magnetic activities. In Sects. 3 and 4 we determine pitch angle and energy diffusion rates due to wave-particle interactions with chorus and plasmaspheric hiss respectively, for extreme values of the plasma density determined from the CRRES measurements using the WAPI code and typical wave characteristics. WAPI, developed at ONERA, is based on quasi linear theory as described in Glauert and Horne (2005) for the PADIE (Pitch Angle Diffusion of Ions and Electrons) code, and calculates fully relativistic quasilinear pitch-angle and energy diffusion coefficients for resonant wave-particle interactions in magnetized plasma. In Sect. 5 the resulting diffusion coefficients are introduced into a physics-based model of the radiation belts, Salammbô-3D (Beutier and Boscher, 1995; Bourdarie, 1996; Varotsou et al., 2005, 2008), to compare the time evolution of energetic electron flux for extreme values of plasma density. Finally, in Sect. 6, the results are discussed and the effects of extreme plasma density values on diffusion rates and on electron fluxes in the radiation belts are examined.

It is important to note that $L$ and $L^{*}$ used in this paper represent, respectively, the McIlwain $L$ parameter and $L$-shell value calculated with ONERA IRBEM library (http://craterre.onecert.fr/prbem/irbem/description.html).

\section{CRRES density distribution}

The Combined Release and Radiation Effects Satellite (CRRES) was launched on 25 July 1990 into a highly elliptical geosynchronous transfer orbit, with a perigee of $350 \mathrm{~km}$, an apogee of $33584 \mathrm{~km}$ and inclination of $18.1^{\circ}$. The orbital period was approximately $10 \mathrm{~h}$, and the initial apogee was at a 
MLT of 08:00 MLT. The magnetic local time of apogee decreased at a rate of approximately $1.3 \mathrm{~h}$ per month until the satellite failed on 11 October 1991, when its apogee was at about 14:00 MLT. This orbit gives good MLT coverage inside $L^{*}=4$ but at $L^{*}=6$ there is a gap in the pre-noon sector that reduces the statistics in the 09:00-12:00 MLT sector. The satellite swept through the heart of the radiation belts on average approximately 5 times per day and provided good coverage of this important region. The plasma density was derived from measurements of the wave electric field in the frequency range $100 \mathrm{~Hz}-400 \mathrm{kHz}$ by the Plasma Wave Experiment (PWE) (Anderson et al., 1992). In the plasmasphere the plasma density was determined from emissions at the upper hybrid frequency. Further out, in the trough region, where the upper hybrid line is usually not well defined, the plasma density was estimated from the lower frequency limit of the electromagnetic continuum radiation, which is taken to be a plasma wave cutoff at the plasma frequency (Gurnett and Shaw, 1973).

Plasma densities derived from the CRRES PWE data have already been studied by Sheeley et al. (2001) and used to define a model of electron plasma density between $L=3$ and $L=7$. In this study, the goal is not to define a new model but rather to assess how variations in the plasma density can affect diffusion rates from wave-particle interactions and the resulting electron distribution in the radiation belts.

In order to study the effect of plasma density on diffusion rates for interaction between energetic particles and waves (chorus and plasmaspheric hiss), we study the distribution of plasma density at representative $L^{*}$ values: $L^{*}=2.5 \pm 0.1$, $L^{*}=4.0 \pm 0.1$ and $L^{*}=6.0 \pm 0.1$, for two MLT intervals, around noon and midnight: $9 \mathrm{~h}<$ MLT $<15 \mathrm{~h}(\sim$ MLT $12 \mathrm{~h})$ and $21 \mathrm{~h}<$ MLT $<3 \mathrm{~h}(\sim$ MLT $0 \mathrm{~h})$ and for three levels of magnetic activity which we define as quite $(\mathrm{Kp}<2)$, moderate $(2 \leq \mathrm{Kp}<4)$ and active $(\mathrm{Kp} \geq 4)$. The probability and cumulative distribution functions of the electron plasma density for these cases are shown in Figs. 1 and 2, respectively. The electron density can vary with latitude, particularly at high latitudes (e.g., Denton et al., 2006). However, near the equator CRRES observations show that the electron density can be fairly flat and not vary significantly with latitude (Denton et al., 2006). In this study we restrict the analysis to equatorial measurements made with $\pm 15^{\circ}$ of the magnetic equator. In Figs. 1 and 2, the electron density provided by three existing models, GCPM (Gallagher et al., 2000), Sheeley model (Sheeley et al., 2001) and Carpenter model (Carpenter and Anderson, 1992), are represented by the dashed, dash-dot and dash-dot-dot-dot lines, respectively. For GCPM, the results come from Gallagher et al. (2000) while for the Carpenter model, the results have been obtained with $R=145$ where $R$ is the 13-month average sunspot number between September 1990 and October 1991, which is the time period of the CRRES measurements. Note that in Sheeley et al. (2001) model, electron density does not depend on magnetic activity, that's why only one red dash-dot lines exists on each plot for this model. Moreover, this model is valid only between $L=3$ and $L=7$.

At $L^{*}=2.5$, Figs. 1 and 2 show that the density distribution does not depend significantly on MLT or magnetic activity. It is well known that the plasmapause location depends on magnetic activity and becomes closer to the Earth as the magnetic activity increases. This implies that some $L^{*}$ values, can be inside or outside the plasmasphere according to magnetic activity. At $L^{*}=2.5$ the observed density distribution is too narrow to characterize the transition inside/outside plasmasphere. Thus, CRRES measurements show that this $L^{*}$ value is predominantly inside the plasmasphere whatever the level of magnetic activity and MLT. However, the dynamics of the electron density in this region is still significant with a variation of more than one decade, between 100 and $2000 \mathrm{~cm}^{-3}$. It seems that this dynamics is a real variability inside the plasmasphere. This dynamics corresponds to the strong seasonal and longitudinal variation shown in Clilverd et al. (2007) at this $L^{*}$ value. Regarding to GCPM and Carpenter model at this $L^{*}$ value, Figs. 1 and 2 show that results provided by these two models are similar, do not depend on magnetic activity or MLT and correspond to the higher measurements of CRRES ( $\sim 95$ th percentile).

At $L^{*}=4.0$, there is much more variability since here the measurements are sometimes inside and sometimes outside the plasmasphere. Figures 1 and 2 show that the electron density depends significantly on magnetic activity with higher density during quiet activity. The large variability of the plasma density reflects in part a real dynamics of electron density inside and outside the plasmasphere but is biased by the plasmapause crossing. At this $L^{*}$ value the densities given by the GCPM, Sheeley model and Carpenter model are very different. From the Carpenter model, for quiet and moderate activity, the electron density is high (inside plasmasphere) and corresponds to the 98th percentile of CRRES data while for active conditions the electron density is lower (outside plasmasphere) and corresponds to the 40th percentile at midnight and to the 60th percentile at noon. From GCPM, for quiet conditions the electron density corresponds to the 95th and 60th percentiles for noon and midnight respectively, for moderate conditions the density corresponds to the 95th and 15th percentiles for noon and midnight and finally for active conditions the electron density is lower than the minimum value measured by CRRES for noon and corresponds to the 1st percentile for midnight. From the Sheeley model, the electron density, with no dependence on magnetic activity, is between the electron density from Carpenter model and the one from GCPM for quiet conditions $(\mathrm{Kp}<2)$.

At $L^{*}=6.0$, Figs. 1 and 2 show that the density distribution depends on magnetic activity but less than at $L^{*}=4.0$. However, there is a big difference in this dependence between noon and midnight: the electron density increases with increasing $\mathrm{Kp}$ at noon but decreases with increasing $\mathrm{Kp}$ at midnight. Concerning GCPM, Sheeley model and Carpenter models, at noon, the densities resulting from GCPM and 

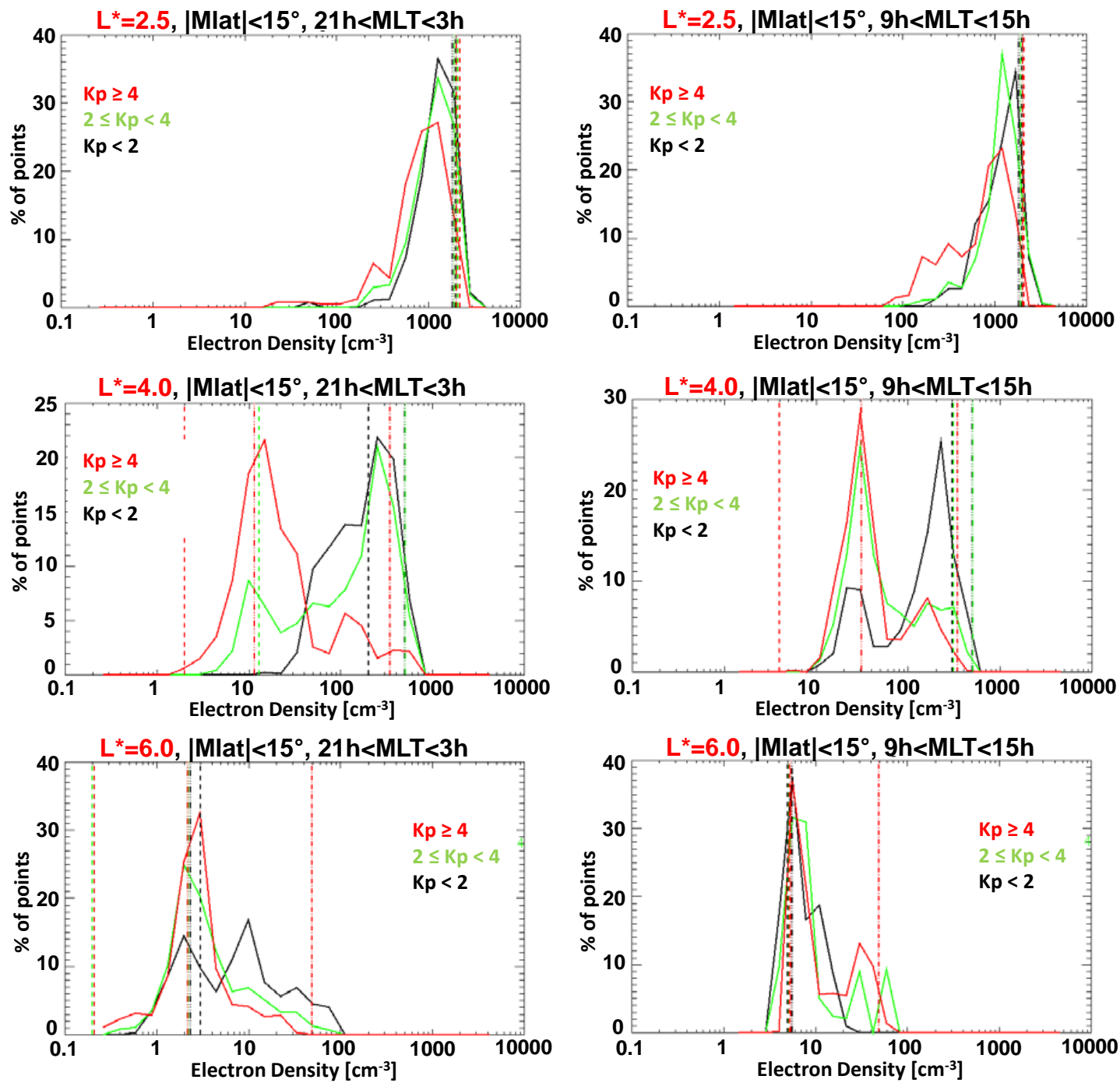

Figure 1. Electron plasma density $\left(\mathrm{cm}^{-3}\right)$ resulting from CRRES measurements versus percentage of points for three $L^{*}$ values $\left(L^{*}=2.5\right.$ on top, $L^{*}=4.0$ in the middle and $L^{*}=6.0$ at the bottom), for two MLT intervals (around midnight on the left and around noon on the right). On each plot, three magnetic activities are represented: quiet (in black), moderate (in green) and active (in red). Results from the GCPM , Sheeley and Carpenter models are also represented in dashed lines, dash-dot lines and dash-dot-dot-dot lines respectively.

Carpenter models are in agreement and correspond to the 40th percentile whatever the magnetic condition, while electron density resulting from Sheeley model is by a factor of 10 higher. At midnight, densities resulting from Carpenter model are the same whatever the magnetic condition and correspond to the 30th percentile for quiet activity, and the 50th percentile for moderate and high activities. The density provided by the GCPM is in agreement with Carpenter results for quiet activity but is really low for moderate and high activity with a value lower than the minimum density measured by CRRES. Electron density resulting from Sheeley model is higher by a factor of ten with Carpenter model, whatever the magnetic conditions at midnight and higher by a factor of one hundred with GCPM model for moderate and active conditions.
Finally, CRRES measurements show that electron density distribution can be very broad with densities covering a range of more than a factor of 100 for $L^{*}$ values close to the plasmapause location $\left(L^{*} \sim 4.0\right)$, depending on magnetic activity. Moreover, other variations, not due to the plasmapause crossing, exists inside $\left(L^{*} \sim 2.5\right)$ and outside the plasmasphere $\left(L^{*} \sim 6.0\right)$, where the distribution is very wide (width by a factor of 10 nearly). This dynamics is somewhat dependent on MLT and magnetic activity. Secondly, the electron density resulting from Carpenter model, Sheeley model and GCPM are not always consistent with each other and with CRRES measurements. The difference in the definition of the plasmapause location is usually the reason of the disagreement. Carpenter model is based on data from ISEE spacecraft. Density values resulting from these data have been 

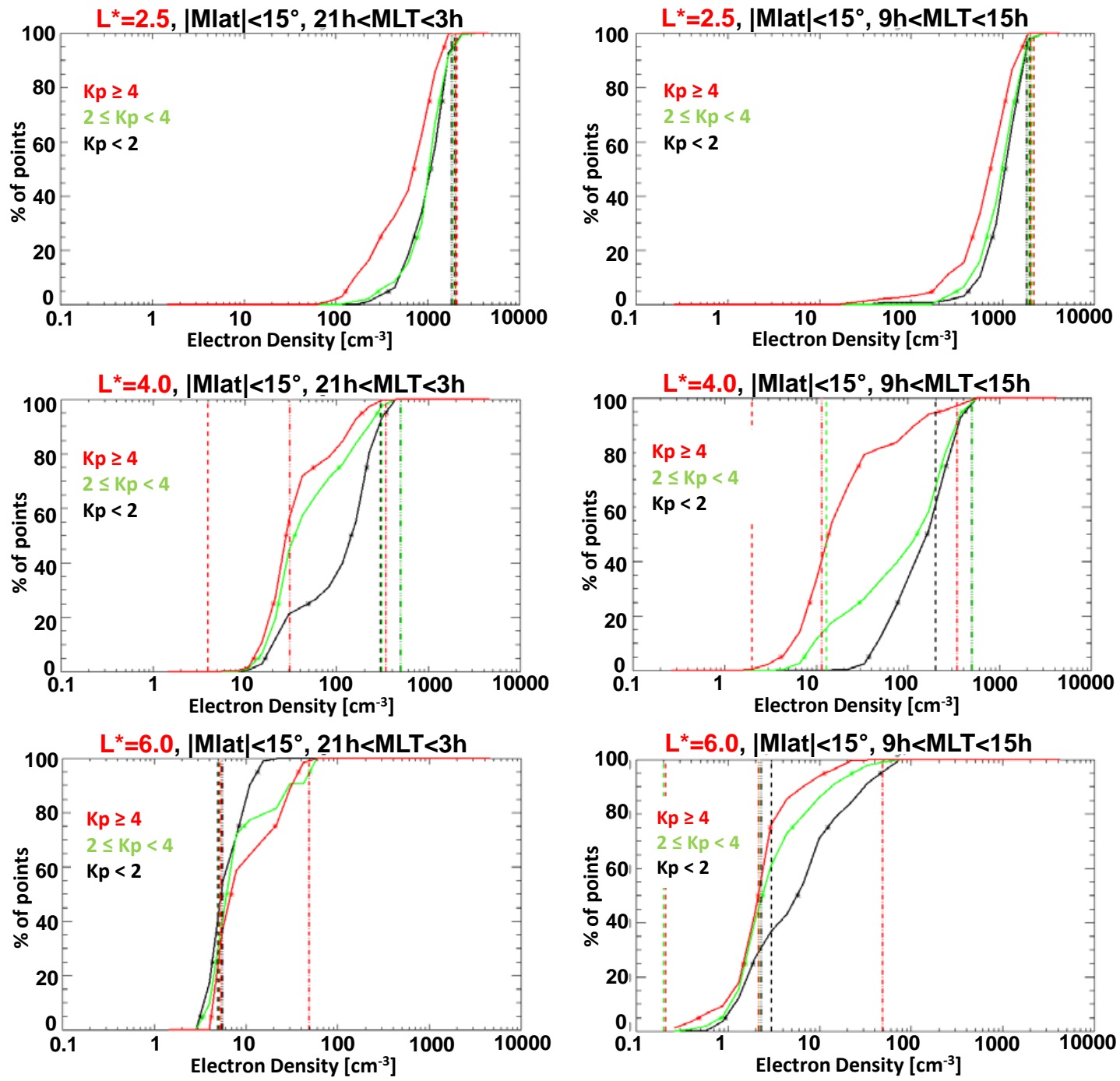

Figure 2. Electron plasma density $\left(\mathrm{cm}^{-3}\right)$ resulting from CRRES measurements versus percentage of cumulated points for three $L^{*}$ values ( $L^{*}=2.5$ on top, $L^{*}=4.0$ in the middle and $L^{*}=6.0$ at the bottom), for two MLT intervals (around midnight on the left and around noon on the right). On each plot, three magnetic activities are represented: quiet (in black), moderate (in green) and active (in red). Results from the GCPM , Sheeley and Carpenter models are also represented in dashed lines, dash-dot lines and dash-dot-dot-dot lines, respectively.

analyzed and a definition of the plasmapause has been defined by fitting the data. For GCPM model it is more complicated: this model uses a modified version of Carpenter and Anderson's (1992) equation to describe the equatorial electron densities by using the measurements from the DE1/RIMS instrument. Then, an exponential function is used by the author to interpolate between the topside ionosphere profile form IRI model (Bilitza and Reinnisch, 2008) and the plasmaspheric density profile (Gallagher et al., 2000).

After the description of the electron plasma density distribution according to $L^{*}$, MLT and magnetic activity and the comparison with existing models, extreme density values, corresponding to the 5th (low density) and the 95th (high density) percentile of distribution plotted in Fig. 2 have been used to compute diffusion rates resulting from wave-particle interactions for chorus and plasmaspheric hiss.

\section{Effect of density dynamics on particle interaction with chorus waves}

To examine the effects of extreme densities, to see what might happen during extreme events, pitch angle and energy diffusion rates have been calculated using the ONERA WAPI code and typical chorus wave parameters. The WAPI code, developed at ONERA, computes pitch angle and energy diffusion coefficients due to wave-particle interaction for all energies and all location in space. WAPI is valid in the case of interactions of electrons and protons with "whistler" 

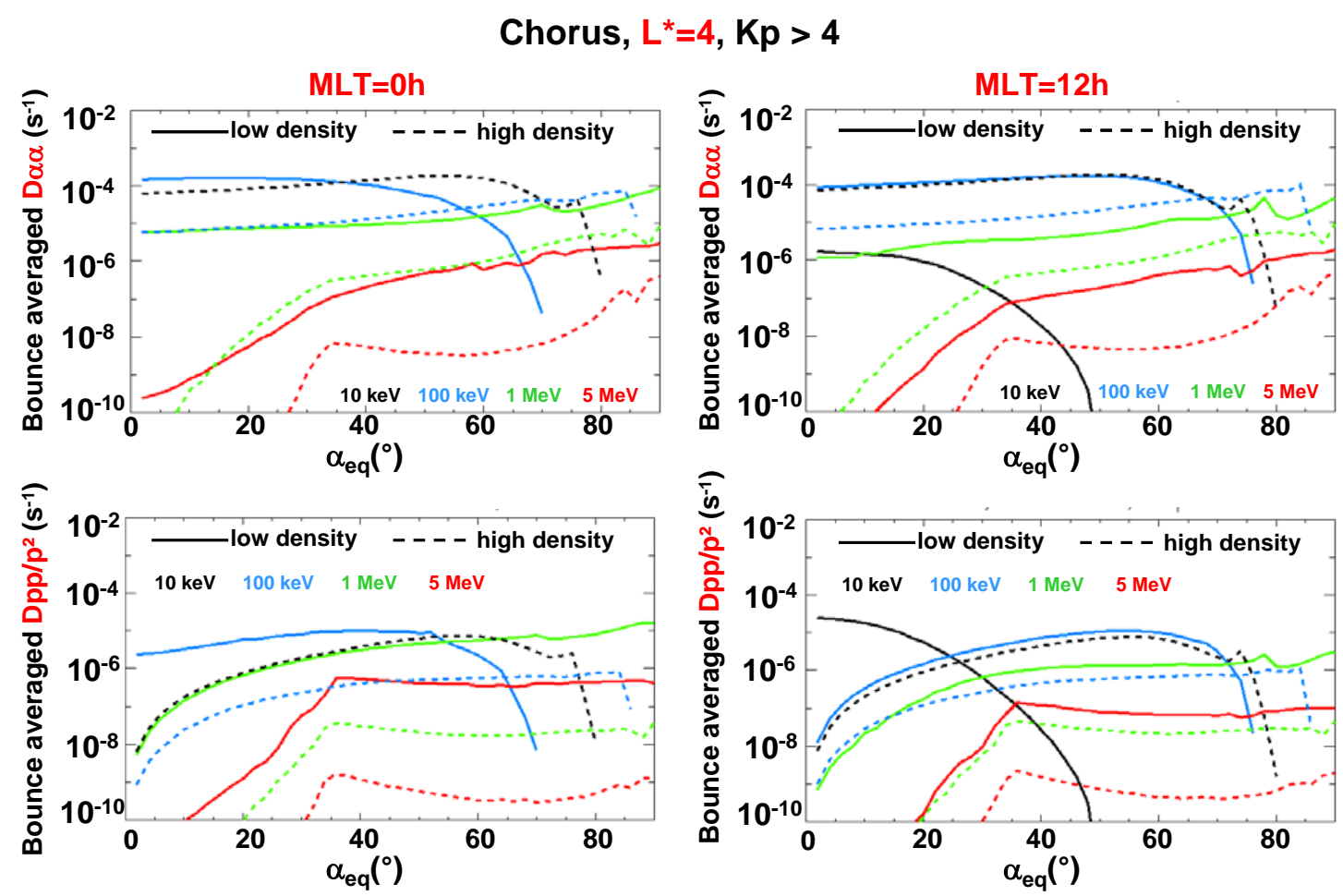

Figure 3. Bounce-averaged pitch angle (top) and energy (bottom) diffusion rates $\left(\mathrm{s}^{-1}\right.$ ) for chorus using WAPI code for 4 energies, $0.01 \mathrm{MeV}$ (black), $0.1 \mathrm{MeV}$ (blue), $1 \mathrm{MeV}$ (green) for and $5 \mathrm{MeV}$ (red) $L^{*}=4$ and for MLT $=0 \mathrm{~h}$ on the left and MLT $=12 \mathrm{~h}$ on the right for active magnetic activity $(\mathrm{Kp}>4)$ and for two different plasma densities: 5 th percentile in solid lines and 95th percentile in dashed lines.

and EMIC waves, in a cold magnetized plasma, whatever the plasma density, the propagation angle and the number of resonances. WAPI uses the quasi-linear theory defined by Kennel et al. (1966), and the diffusion coefficients definition described by Lyons $(1974 a, b)$ and Albert $(1999,2005)$. WAPI code is based on the resolution of two main equations: the dispersion relation and the resonance condition. The numerical techniques used to resolve these equations are similar to the ones described in Glauert and Horne (2005).

The wave parameters used in this study are based on values used by Glauert and Horne (2005), revised using values from fits to data from Horne et al. (2013). We set $f_{\mathrm{m}}=0.3\left|f_{\mathrm{ce}}\right|$, $\delta_{f}=0.1\left|f_{\mathrm{ce}}\right|, f_{\mathrm{lc}}=f_{\mathrm{m}}-2 \delta_{f}, f_{\mathrm{uc}}=f_{\mathrm{m}}+2 \delta_{\mathrm{f}}, X_{\mathrm{m}}=0, X_{\mathrm{w}}=$ $\tan \left(30^{\circ}\right), X_{\min }=0$ and $X_{\max }=\tan \left(45^{\circ}\right)\left(f_{\mathrm{m}}\right.$ and $\delta_{\mathrm{f}}$ are the frequency of maximum wave power and bandwidth, respectively, $f_{\mathrm{lc}}$ and $f_{\mathrm{uc}}$ are lower and upper bounds to the wave spectrum, $X_{\mathrm{w}}$ is the angular width and $X_{\mathrm{m}}$ is the peak of the distribution of wave normal angles, with $X$ the tangent of the wave propagation angle $\varphi$ ). During active conditions multiple satellite observations show that average lower band equatorial chorus amplitudes are typically of the order 25 to $50 \mathrm{pT}$ outside the plasmapause at $L^{*}=4$ and 6 , respectively (Meredith et al., 2012). We choose a representative value of $30 \mathrm{pT}$ up to $\left|\lambda_{\mathrm{m}}\right|=30^{\circ}$, and zero beyond. The Landau resonance $n=0$ and \pm 5 cyclotron resonances are included in the calculations. Chorus waves are mainly localized between
MLT $=0 \mathrm{~h}$ and MLT $=12 \mathrm{~h}$, so that diffusion rates for chorus waves have been calculated at two magnetic longitudes: $\mathrm{MLT}=0 \mathrm{~h}$ and $\mathrm{MLT}=12 \mathrm{~h}$.

A general expression for the diffusion of plasma particles by resonant wave-particle interactions using quasilinear theory was derived by Kennel and Engelmann (1966) and can be represented by three parameters: the diffusion coefficients in pitch angle D $\alpha \alpha$, momentum Dpp, and mixed pitch angle-momentum Dap. In the next figures, diffusion coefficients are plotted versus equatorial pitch angle of electron, which represents the angle of speed vector of electron with magnetic field vector at equator. Figures 3 and 4 show the bounce-averaged pitch angle D $\alpha \alpha$ (top) and momentum $\mathrm{Dpp} / \mathrm{p}^{2}$ (bottom) diffusion rates $\left(\mathrm{s}^{-1}\right)$ for chorus waves using the WAPI code for 4 energies, $0.01 \mathrm{MeV}$ (black), $0.1 \mathrm{MeV}$ (blue), $1 \mathrm{MeV}$ (green) and $5 \mathrm{MeV}$ (red) for MLT $=0 \mathrm{~h}$ on the left and MLT $=12 \mathrm{~h}$ on the right for active magnetic conditions $(\mathrm{Kp}>4)$ for two different plasma densities: 5th percentile (low density) in solid lines and 95th percentile (high density) in dashed lines for $L^{*}=4.0$ (Fig. 3) and $L^{*}=6.0$ (Fig. 4). These figures show the big influence of extreme plasma density on pitch angle and momentum diffusion rates for chorus waves. However, several different types of behaviour can be observed according to energy, $L^{*}$ and MLT. Note that wave-particle interaction is controlled by two equations: the resonance equation and the dispersion 

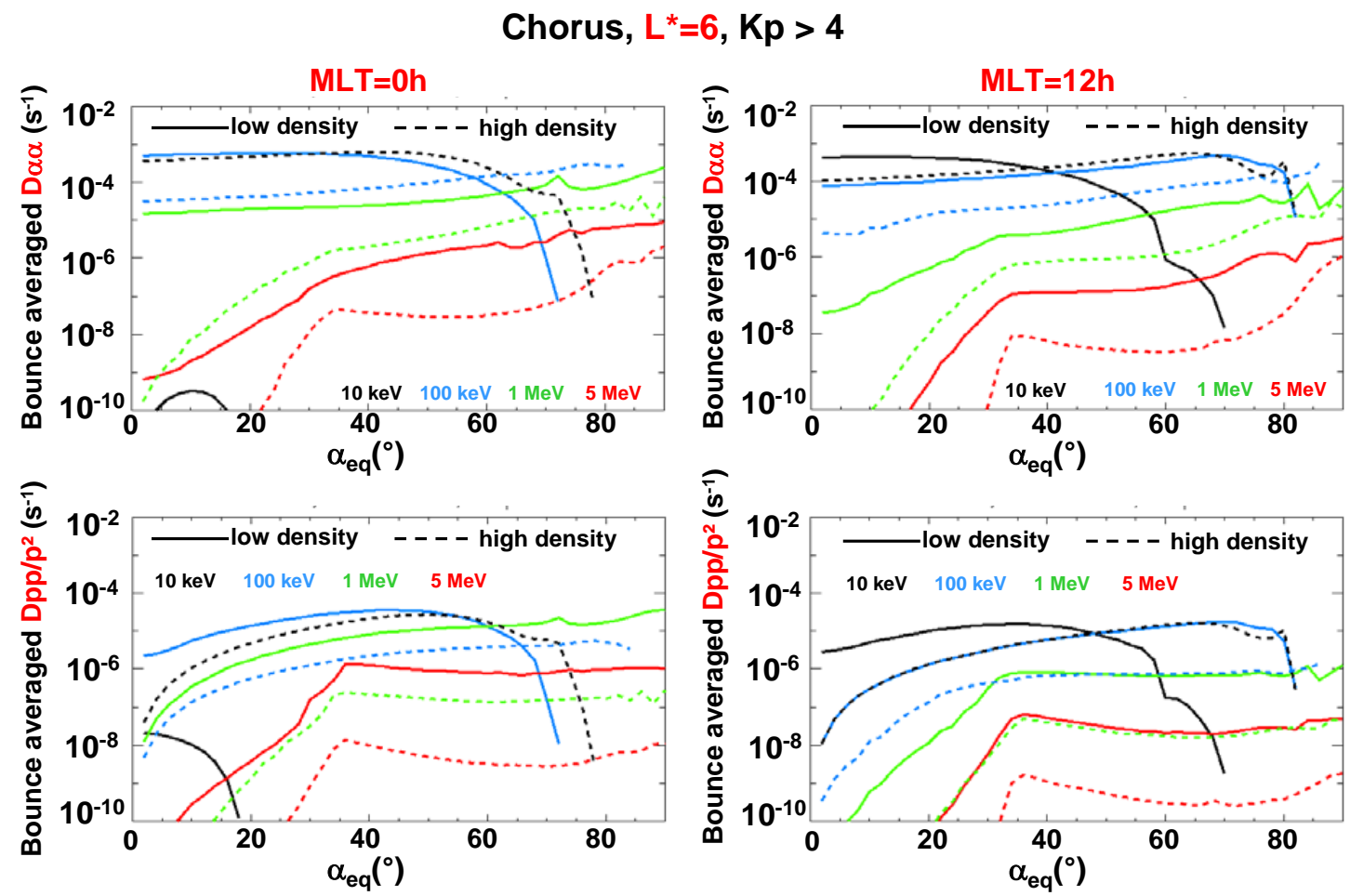

Figure 4. Bounce-averaged pitch angle (top) and energy (bottom) diffusion rates ( $\mathrm{s}^{-1}$ ) for chorus using WAPI code for 4 energies, $0.01 \mathrm{MeV}$ (black), $0.1 \mathrm{MeV}$ (blue), $1 \mathrm{MeV}$ (green) for and $5 \mathrm{MeV}$ (red) $L^{*}=6$ and for MLT $=0 \mathrm{~h}$ on the left and MLT $=12 \mathrm{~h}$ on the right for active magnetic activity $(\mathrm{Kp}>4)$ and for two different plasma densities: 5 th percentile in solid lines and 95th percentile in dashed lines.

relation. Thus, the differences in behaviour of diffusion rates are due to the fact that the change in density changes the dispersion relation for the waves and thus changes the resonant energy. According to a given density value and a given location, waves will interact with a given energy and this energy changes when the density changes.

At low energy ( $10 \mathrm{keV}$ : black lines) the increase of plasma density leads to an increase of $\mathrm{D} \alpha \alpha$ and $\mathrm{Dpp} / \mathrm{p}^{2}$ of several decades for all pitch angles whatever MLT and $L^{*}$ except for small pitch angles at noon where $\mathrm{Dpp} / \mathrm{p}^{2}$ and both $\mathrm{D} \alpha \alpha$ and $\mathrm{Dpp} / \mathrm{p}^{2}$ decrease with increasing density at $L^{*}=4$ and $L^{*}=6$, respectively.

At higher energy $(\geq 100 \mathrm{keV})$, the behaviour of the diffusion rates is different to that at low energy and is the same whatever $L^{*}$ and MLT. Both $\mathrm{D} \alpha \alpha$ and Dpp/p $\mathrm{p}^{2}$ decrease while density increases, except at large pitch angle for $100 \mathrm{keV}$ electrons. The behaviour for $100 \mathrm{keV}$ electrons seems to be a transition between the behaviour at very low energy and the ones at higher energy.

For any given diffusion coefficient the magnitude of the decrease is pitch angle dependent with the largest decrease at small pitch angles.

These simulations show that the dynamics of the electron plasma density has a significant influence on the pitch angle and energy diffusion rates due to wave particle interactions between chorus waves and energetic electrons. During extreme events, the variation in the diffusion coefficients using the two extreme electron density values (5th and 95th percentiles) can reach up to 6 decades for a given MLT and a given $L^{*}\left(\mathrm{MLT}=0 \mathrm{~h}\right.$ and $L^{*}=6.0$ for low energy). These results confirm that the electron plasma density plays a significant role in the interactions between chorus and energetic electrons.

However it is important to note that in all the simulations of this study we have neglected the effect of changing background density on the generation and amplification of the waves themselves (Clilverd et al., 2007). This is beyond the scope of the paper and requires further study.

\section{Effect of density dynamics on particle interaction with plasmaspheric hiss}

To examine the effects of extreme density values on the diffusion rates due to plasmaspheric hiss we also calculate the pitch angle and energy diffusion rates due to plasmaspheric hiss using the WAPI code. We set the wave properties of plasmaspheric hiss based on the experimental values determined by Meredith et al. (2007). In this paper the authors show that plasmaspheric hiss may be represented by two Gaussian functions with parameters depending on $L^{*}$ value. Observational (Agapitov et al., 2013) and theoretical studies (Bortnik et al., 2011; Chen et al., 2012a, b) suggest that the 

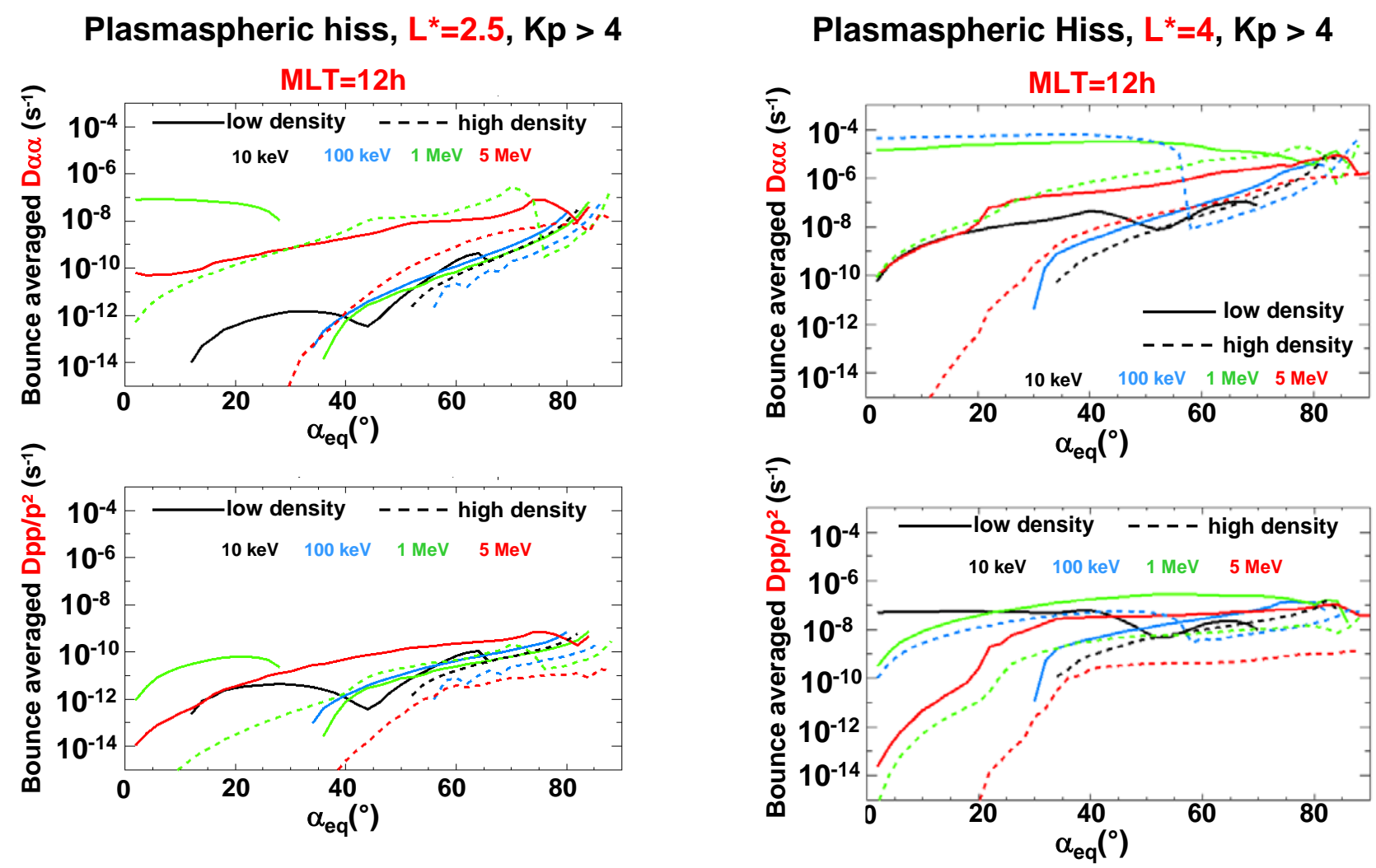

Figure 5. Bounce-averaged pitch angle (top) and energy (bottom) diffusion rates $\left(\mathrm{s}^{-1}\right)$ for plasmaspherc hiss using WAPI code for 4 energies, $0.01 \mathrm{MeV}$ (black), $0.1 \mathrm{MeV}$ (blue), $1 \mathrm{MeV}$ (green) for and $5 \mathrm{MeV}$ (red) $L^{*}=2.5$, for MLT $=0 \mathrm{~h}$, for active magnetic activity $(\mathrm{Kp}>4)$ and for two different plasma densities: 5th percentile in solid lines and 95th percentile in dashed lines.

wave normal angle of plasmaspheric hiss tends to be fieldaligned near the equator and oblique at higher latitudes. Since field-aligned hiss at small or medium wave normal angles is most effective in scattering energetic electrons (Meredith et al., 2006), for the purposes of this study we assume that plasmaspheric hiss is field-aligned with a width of $\tan 20 \mathrm{de}-$ grees. The hiss waves parameters used in this study are summarized in Table 1.

The simulations with WAPI have been performed with a constant wave amplitude, $B_{\mathrm{w}}$, up to $\left|\lambda_{\mathrm{m}}\right|=30^{\circ}$ and zero beyond. The Landau resonance $n=0$ and \pm 5 cyclotron resonances are included in the calculations. Strong plasmaspheric hiss is mainly observed between MLT $=6 \mathrm{~h}$ and MLT $=18 \mathrm{~h}$ (Meredith et al., 2007), so that diffusion rates for plasmaspheric hiss have been calculated only at one magnetic longitude: MLT $=12 \mathrm{~h}$.

Figures 5 and 6 represent the bounce-averaged pitch angle $\mathrm{D} \alpha \alpha$ (top) and momentum Dpp $/ \mathrm{p}^{2}$ (bottom) diffusion rates $\left(\mathrm{s}^{-1}\right)$ for plasmaspheric hiss using the WAPI code for $4 \mathrm{en}$ ergies, $0.01 \mathrm{MeV}$ (black), $0.1 \mathrm{MeV}$ (blue), $1 \mathrm{MeV}$ (green) for and $5 \mathrm{MeV}$ (red), for MLT $=12 \mathrm{~h}$, for active magnetic activity $(\mathrm{Kp}>4)$ for two different plasma densities: 5 th percentile

Figure 6. Bounce-averaged pitch angle (top) and energy (bottom) diffusion rates $\left(\mathrm{s}^{-1}\right)$ for plasmaspheric hiss using WAPI code for 4 energies, $0.01 \mathrm{MeV}$ (black), $0.1 \mathrm{MeV}$ (blue), $1 \mathrm{MeV}$ (green) for and $5 \mathrm{MeV}$ (red) $L^{*}=4.0$, for MLT $=0 \mathrm{~h}$, for active magnetic activity $(\mathrm{Kp}>4)$ and for two different plasma densities: 5th percentile in solid lines and 95th percentile in dashed lines.

(low density) in solid lines and 95th percentile (high density) in dashed lines for $L^{*}=2.5$ (Fig. 5) and for $L^{*}=4$ (Fig. 6). These figures show that the plasma density also has a significant effect on the pitch angle and momentum diffusion rates due to plasmaspheric hiss. However, the diffusion rates resulting from interactions between plasmaspheric hiss and energetic electrons are lower than those resulting from interactions with chorus waves, typically at least 2 orders of magnitude lower.

At low energy $(10 \mathrm{keV})$, there is no big modification in diffusion rates due to the increase of the density. At medium energy $(100 \mathrm{keV})$, the increase of plasma density leads to an increase of diffusion rates for small pitch angle at $L^{*}=4$ while there is no big modification at $L^{*}=2.5$.

For higher energy ( $\geq 1 \mathrm{MeV})$, the increase of plasma density leads to a decrease of diffusion rates, both $\mathrm{D} \alpha \alpha$ and $\mathrm{Dpp} / \mathrm{p}^{2}$, as for chorus.

Finally, the diffusion coefficients show that the dynamics of electron plasma density significantly affects the diffusion rates due to the interaction between plasmaspheric hiss and energetic electrons. However, the diffusion rates due to plasmaspheric hiss are lower than those due to chorus waves so the effect on the energetic electrons will probably be smaller. 
Table 1. Hiss waves parameters used in this study.

\begin{tabular}{lllllllllllllll}
\hline & $\begin{array}{l}f_{\mathrm{m} 1} \\
(\mathrm{~Hz})\end{array}$ & $\begin{array}{l}\delta_{\mathrm{f} 1} \\
(\mathrm{~Hz})\end{array}$ & $\begin{array}{l}f_{\mathrm{lc} 1} \\
(\mathrm{~Hz})\end{array}$ & $\begin{array}{l}f_{\mathrm{uc} 1} \\
(\mathrm{~Hz})\end{array}$ & $\begin{array}{l}B_{\mathrm{w} 1} \\
(\mathrm{pT})\end{array}$ & $\begin{array}{l}f_{\mathrm{m} 2} \\
(\mathrm{~Hz})\end{array}$ & $\begin{array}{l}\delta_{f 2} \\
(\mathrm{~Hz})\end{array}$ & $\begin{array}{l}f_{\mathrm{lc} 2} \\
(\mathrm{~Hz})\end{array}$ & $\begin{array}{l}f_{\mathrm{uc} 2} \\
(\mathrm{~Hz})\end{array}$ & $\begin{array}{l}B_{\mathrm{w} 2} \\
(\mathrm{pT})\end{array}$ & $X_{\mathrm{m}}$ & $X_{\mathrm{w}}$ & $X_{\min }$ & $X_{\max }$ \\
\hline$L^{*}=2.5$ & 293 & 302 & 100 & 883 & 29.6 & 0.1 & 1200 & 883 & 2000 & 4.58 & 0 & $\tan \left(20^{\circ}\right)$ & 0 & $\tan \left(30^{\circ}\right)$ \\
\hline$L=4.0$ & 366 & 450 & 100 & 951 & 27.4 & 0.1 & 1460 & 951 & 2000 & 12 & 0 & $\tan \left(20^{\circ}\right)$ & 0 & $\tan \left(30^{\circ}\right)$ \\
\hline
\end{tabular}

\section{Evolution of the particle fluxes}

It is difficult to estimate the impact of the waves on the radiation belt fluxes from the diffusion coefficients alone since the effects of electron density dynamics depend on energy and pitch angle of the particle and the type of wave for a given $L^{*}$ and MLT. That's why it appears essential to simulate the electron distribution function using the diffusion rates presented above, then convert it into electron fluxes and compare the results using extreme density values (5th and 95th percentiles). To do this, we solved a Fokker Planck equation using the Salammbô code (Beutier and Boscher, 1995; Bourdarie et al., 1996; Varotsou et al., 2005, 2008). Salammbô is a physical model of the Earth's radiation belts, which was developed at ONERA in the 1990s. Salammbô is a three dimensional code (energy, pitch angle, $L^{*}$ ) and gives the electron distribution function anywhere in the radiation belts. In this study, we exclude radial diffusion and focus solely on the interactions of the energetic electrons with the waves and the atmosphere. Mixed pitch angle-energy diffusion coefficients are also neglected. In order to estimate the time evolution of the electron fluxes for the extreme values of the electron density, the fluxes have been calculated at given $L^{*}$ values for particles bouncing near the loss cone. For the initial condition, we use a kappa law, depending on Kp, based on POES data (Sicard-Piet et al., 2011):

$F\left(E, L^{*}\right)=F_{0}\left[1+\frac{E}{T \kappa}\right]^{-(\kappa+1)}$,

with $\kappa=5.9606-0.3595 \mathrm{Kp}$;

$T[\mathrm{keV}]=1.2637-0.0917 \mathrm{Kp}$;

$F_{0}\left[\mathrm{MeV}^{-3} \mathrm{~s}^{-3}\right]=\exp (78.814+0.3661 \mathrm{Kp})$.

Figure 7 shows the time evolution of electron flux due to chorus waves at MLT $=0 \mathrm{~h}$ for $L^{*}=6.0$ and $\mathrm{Kp}>4$ using the two extreme values of the electron plasma density: 5 th percentile on top (low density) and 95th percentile on bottom (high density). The flux is shown at intervals of $6 \mathrm{~h}$. In the low density case (top panel), there is a tipping point at $500 \mathrm{keV}$ with fluxes increasing and decreasing above and below this value respectively. The largest flux increase, of the order of 3 orders of magnitude, is seen at $2 \mathrm{MeV}$. In contrast, the largest flux decrease is of the order of 4 orders of magnitude at $20 \mathrm{keV}$. The entire spectrum approaches a steady state after about $24 \mathrm{~h}$ but the major modification of the spectrum
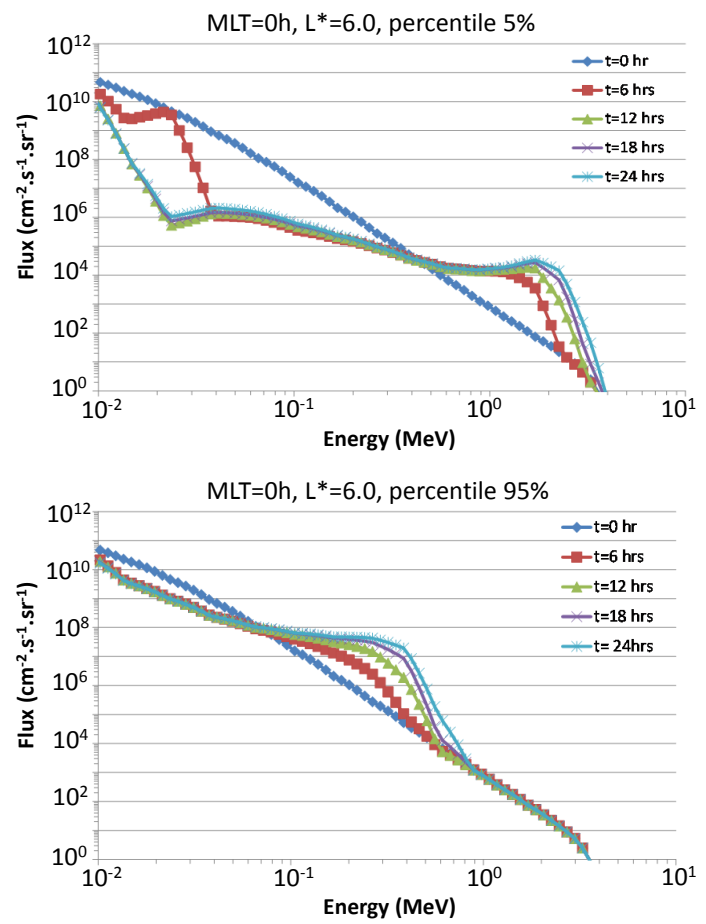

Figure 7. Time evolution of electron flux due to chorus waves at $\mathrm{MLT}=0 \mathrm{~h}$ for $L^{*}=6.0 \mathrm{using}$ extreme electron plasma density values:percentile $5 \%$ on top (low density) and percentile $95 \%$ on bottom (high density). The flux is shown at interval of $6 \mathrm{~h}$.

is during the first $6 \mathrm{~h}$. These timescales are comparable to the observed timescale for flux increase in the radiation belts during a magnetic storm (Baker et al., 1986, 1994; Kim and Chan, 1997; Meredith et al., 2002a, b). For high density (bottom panel), the behaviour of the spectrum is markedly different. The tipping point is at a lower energy, around $80 \mathrm{keV}$. The largest flux increase in this case, of 3 orders of magnitude, is seen at $400 \mathrm{keV}$ and there is no acceleration above $800 \mathrm{keV}$.

Thus, there is a shift in energy in the increase of flux between the simulations using low and high density. The simulation with low density leads to an acceleration of electrons with significantly higher energy than the simulation with high density. Moreover, in the simulation using high density, the decrease of flux, for electron $<80 \mathrm{keV}$, is minor comparing to the decrease in the low density simulation. 

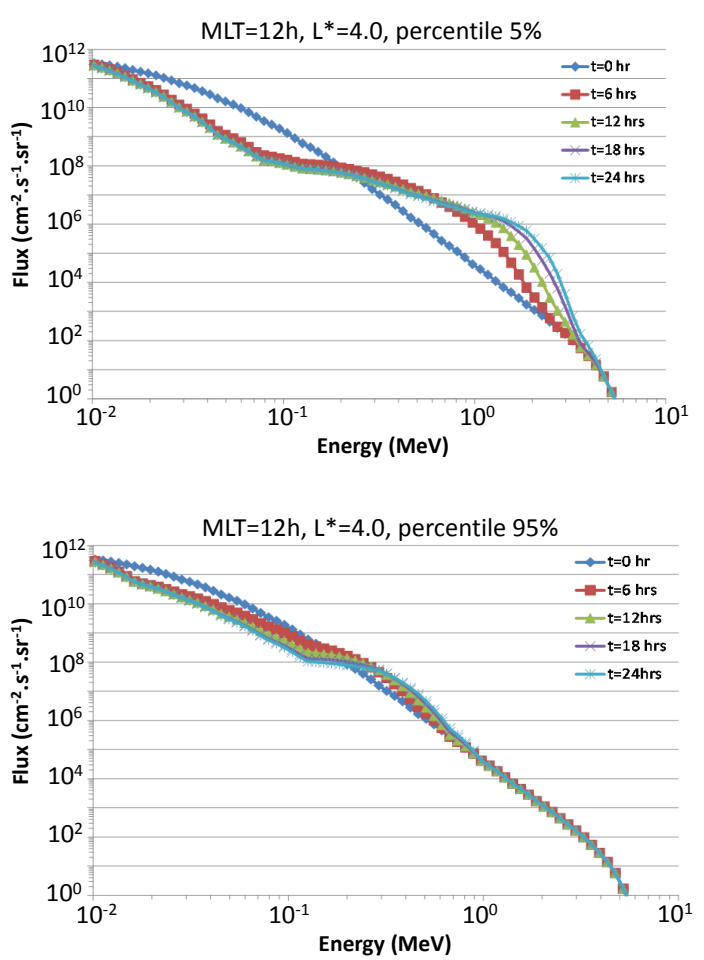

Figure 8. Time evolution of electron flux due to chorus and hiss waves at MLT $=12 \mathrm{~h}$ for $L^{*}=4.0$ using extreme electron plasma density values: percentile $5 \%$ on top (low density) and percentile $95 \%$ on bottom (high density). The flux is shown at interval of $6 \mathrm{~h}$.

Figure 8 shows the time evolution of electron flux due to chorus and plasmaspheric hiss at MLT $=12 \mathrm{~h}$ for $L^{*}=4.0$ and $\mathrm{Kp}>4$ using extreme electron plasma density values: 5th percentile on top (low density) and 95th percentile on bottom (high density). In the low density case (top panel), there is a tipping point at $250 \mathrm{keV}$ with fluxes increasing and decreasing above and below this value respectively. The largest flux increase, of the order of 3 orders of magnitude, is again seen near $2 \mathrm{MeV}$. Below the tipping point the fluxes decrease by about a decade for energies in the range 30 $100 \mathrm{keV}$. The simulation using high density (bottom panel) leads to a minor effect on electron fluxes: acceleration and losses are weak.

\section{Discussion}

Energetic electrons in the Earth's radiation belts circle the globe in minutes to hours depending on electron energy and may interact with plasma waves at all local times. In their recent modelling of a rapid acceleration event by whistler mode chorus observed by the Van Allen probes, Thorne et al. (2013) determined the plasma density directly from Van Allen probe data in the region 04:00-12:00 MLT, but had to revert to a model from 12:00-24:00 and 00:00-04:00 MLT. In this region they estimated the plasma density from the
Sheeley et al. model (2001) scaled down by a factor of 3.5 based on the satellite measurements in the 04:00-12:00 sector. This study emphasizes the need for accurate quantification of the plasma density as a function of spatial location and magnetic activity for radiation belt modelling.

Our study shows that variations of the electron plasma density are significant. According to CRRES measurements, the electron plasma density can vary up to a factor 100 at given $L^{*}$, MLT and $\mathrm{Kp}$, inside or outside plasmasphere. The dynamics can even be higher around $L^{*}=4.0$ in the plasmapause region due to the crossing of the plasmapause.

The goal of this study was to estimate the influence of such dynamics on the diffusion rates due to interaction between energetic particles and waves (chorus and plasmaspheric hiss) and ultimately on electron flux in the radiation belts.

Using extreme values of the plasma density (5th and 95th percentiles), the pitch angle and energy diffusion coefficients were calculated and compared for several $L^{*}$ in the case of chorus and plasmaspheric hiss. The dynamics of electron plasma density leads to a large variation of both pitch angle and energy diffusion coefficients. The difference between the diffusion coefficients determined using low density and high densities can reach 6 decades, particularly at small equatorial pitch angles, near the loss cone. The results confirm that electron plasma density is a key parameter in the wave-particle interaction modelling. Thus, the electron plasma density needs to be defined accurately inside and outside plasmasphere, as well as in the plasmapause region.

To evaluate the effect of electron plasma density dynamics on energetic electron flux in the radiation belts, we used Salammbô to estimate the time evolution of the energetic electron flux. The results confirm that wave-particle interactions lead to an increase of flux for energetic electrons greater than few tens of keV and a decrease of flux for lower energy. However a big difference exists in the time evolution of electron flux according to high or low electron plasma density values used in the wave-particle interaction: acceleration and loss of electron are most efficient using low electron plasma density. It is well known that wave acceleration is efficient in low density region (outside plasmapause) (Horne et al., 2005) but this study shows that there is a difference in this region in the time evolution of electron flux due to lowest density values or highest density values.

\section{Conclusions}

We have used measurements of the plasma density derived from the CRRES-PWE to determine extreme values of the plasma density. We then assessed the role of extreme plasma densities on the diffusion rates and the energetic electron flux. Our principle results are as follows: 
1. The highest densities are observed near $L^{*}=2.5$ while the lowest densities occur near $L^{*}=6$. The largest variability in the distributions occurs near $L^{*}=4$.

2. The plasma density dynamics plays an important role on diffusion rates due to wave-particle interactions: for low energy electrons ( $\leq 100 \mathrm{keV}) \mathrm{D} \alpha \alpha$ and Dpp typically increase with increasing density, whereas, for higher energy electrons, $(\geq 1 \mathrm{MeV}) \mathrm{D} \alpha \alpha$ and Dpp typically decrease by several orders of magnitude with increasing density. For electrons with energy between $100 \mathrm{keV}$ and $1 \mathrm{MeV}$, the effect of density on diffusion coefficient is no so clear: it is a transition between the behaviour at low energy and the one at high energy. Thus $\mathrm{D} \alpha \alpha$ and Dpp sometimes decrease with increasing density but sometimes increase according to MLT or $L^{*}$ values.

3. Using the Salammbô model and diffusion rates calculated with WAPI we show that at $L^{*}=6$, in the low density case, using the density from the 5 th per centile, electron energy diffusion by chorus waves is strongest at $2 \mathrm{MeV}$ and increases the flux by up to 3 orders of magnitude over a period of $24 \mathrm{~h}$.

4. In the high density case, using the density from the 95th percentile, there is little acceleration at energies above $800 \mathrm{keV}$ at $L^{*}=6$, and virtually no acceleration at $L^{*}=4$. In the high density case the strongest energy diffusion occurs at lower energies around $400 \mathrm{keV}$ where the flux at $L^{*}=6$ increases 3 orders of magnitude.

The results show that accurate quantification of the plasma density as a function of spatial location and magnetic activity is essential for dynamic radiation belt modelling.

Acknowledgements. We acknowledge Roger Anderson for provision of the CRRES plasma wave data. The research leading to these results has received funding from the European Union Seventh Framework Programme (FP7/2007-2013) under grant agreement 262468 (SPACECAST) and the UK Natural Environment Research Council. Finally, we especially thank the French Space Agency (CNES) for their support for several years on the development of WAPI code.

Topical Editor G. Balasis thanks two anonymous referees for their help in evaluating this paper.

\section{References}

Abel, B. and Thorne, R. M.: Electron scattering loss in Earth's inner magnetosphere: 1. Dominant physical processes, J. Geophys. Res., 103, 2385-2396, doi:10.1029/97JA02919, 1998a.

Abel, B. and Thorne, R. M.: Electron scattering loss in Earth's inner magnetosphere: 2. Sensitivity to model parameters, J. Geophys. Res., 103, 2397-2407, doi:10.1029/97JA02920, 1998 b.
Agapitov, O., Artemyev, A., Krasnoselskikh, V., Khotyaintsev, Y. V., Mourenas, D., Breuillard, H., Balikhin, M., and Rolland, G.: Statistics of whistler-mode waves in the outer radiation belt: Cluster STAFF-SA measurements, J. Geophys. Res. Space Phys., 118, 3407-3420, doi:10.1002/jgra.50312, 2013.

Albert, J. M.: Analysis of quasi-linear diffusion coefficients, J. Geophys. Res., 104, 2429-2441, 1999.

Albert, J. M.: Evaluation of quasi-linear diffusion coefficients for whistler mode waves in a plasma with arbitrary density ratio, J. Geophys. Res., 110, A03218, doi:10.1029/2004JA010844, 2005.

Albert, J. M., Meredith, N. P., and Horne, R. B.: Three-dimensional diffusion simulation of outer radiation belt electrons during the October 9, 1990, magnetic storm, J. Geophys. Res., 114, A09214, doi:10.1029/2009JA014336, 2009.

Anderson, R. R., Gurnett, D. A., and Odem, D. L.: CRRES plasma wave experiment, J. Spacecr. Rockets, 29, 570-573, 1992.

Artemyev, A. V., Orlova, K. G., Mourenas, D., Agapitov, O. V., and Krasnoselskikh, V. V.: Electron pitch-angle diffusion: resonant scattering by waves vs. nonadiabatic effects, Ann. Geophys., 31, 1485-1490, doi:10.5194/angeo-31-1485-2013, 2013.

Baker, D. N., Blake, J. B., Klebesadel, R. W., and Higbie, P. R.: Highly relativistic electrons in the Earth's outer magnetosphere: 1. Lifetimes and temporal history 1979-1984, J. Geophys. Res., 91, 4265-4276, doi:10.1029/JA091iA04p04265, 1986.

Baker, D. N., Blake, J. B., Callis, L. B., Cummings, J. R., Hovestadt, D., Kanekal, S., Klecker, B., Mewaldt, R. A., and Zwickl, R. D.: Relativistic electron acceleration and decay time scales in the inner and outer radiation belts: SAMPEX, Geophys. Res. Lett., 21, 409-412, doi:10.1029/93GL03532, 1994.

Beutier, T. and Boscher, D.: A three-dimensional analysis of the electron radiation belt by the Salammbô code, J. Geophys. Res., 100, 14853-14861, doi:10.1029/94JA03066, 1995.

Bilitza, D. and Reinisch, B. W.: International Reference Ionosphere 2007: Improvements and new parameters, Adv. Space Res., 42, 599-609, doi:10.1016/j.asr.2007.07.048, 2008.

Bortnik, J. and Thorne, R. M.:. The dual role of ELF/VLF chorus waves in the acceleration and precipitation of radiation belt electrons, J. Atmos. Sol. Terr. Phys., Special Issue: Global Aspects of Magnetosphere-Ionosphere Coupling, 67, 378-386, 2007.

Bortnik, J., Chen, L., Li, W., Thorne, R. M., and Horne, R. B.: Modeling the evolution of chorus waves into plasmaspheric hiss, J. Geophys. Res., 116, A08221, doi:10.1029/2011JA016499, 2011.

Bortnik, J., Thorne, R. M., and Meredith, N. P.: The unexpected origin of plasmaspheric hiss from discrete chorus emissions, Nature 452, 62-66, doi:10.1038/nature06741, 2008.

Boscher, D., Bourdarie, S., Lazaro, D., and Sicard-Piet, A.: Technical report for CNES study, RF 2/12841 DESP, 2007.

Bourdarie, S., Boscher, D., Beutier, T., Sauvaud, J.-A., and Blanc, M.: Magnetic storm modeling in the Earth's electron belt by the Salammbô code, J. Geophys. Res., 101, 27171-27176, doi:10.1029/96JA02284, 1996.

Carpenter, D. L. and Anderson, R. R.: An ISEE/whistler model of equatorial electron density in the magnetosphere, J. Geophys. Res., 97, 1097-1108, doi:10.1029/91JA01548, 1992.

Chasovitin, Y. K., Gulyaeva, T. L., Deminov, M. G., and Ivanova, S. E.: Russian Standard Model of Ionosphere (SMI), in: COST251TD (98)005, edited by: Vernon, A., 161-172, Rutherford Appleton Lab., Didcot, UK, 1998. 
Chen, L., Bortnik, J., Li, W., Thorne, R. M., and Horne, R. B.: Modeling the properties of plasmaspheric hiss : 1. Dependence on chorus wave emission, J. Geophys. Res., 117, A05201, doi:10.1029/2011JA017201, 2012a.

Chen, L., Li, W., Bortnik, J., and Thorne, R. M.: Amplification of whistler-mode hiss inside the plasmasphere, Geophys. Res. Lett., 39, L08111, doi:10.1029/2012GL051488, 2012b.

Clilverd, M. A., Meredith, N. P., Horne, R. B., Glauert, S. A., Anderson, R. R., Thomson, N. R., Menk, F. W., and Sandel, B. R.: Longitudinal and seasonal variations in plasmaspheric electron density: Implications for electron precipitation, J. Geophys. Res., 112, A11210, doi:10.1029/2007JA012416, 2007.

Denton, R. E., Menietti, J. D., Goldstein, J., Young, S. L., and Anderson, R. R.: Electron density in the magnetosphere, J. Geophys. Res., 109, A09215, doi:10.1029/2003JA010245, 2004.

Denton, R. E., Takahashi, K., Galkin, I. A., Nsumei, P. A., Huang, X., Reinisch, B. W., Anderson, R. R., Sleeper, M. K., and Hughes, W. J.: Distribution of density along magnetospheric field lines, J. Geophys. Res., 111, A04213, doi:10.1029/2005JA011414, 2006.

Fok, M.-C., Horne, R. B., Meredith, N. P., and Glauert, S. A.: Radiation Belt Environment Model: Application to space weather nowcasting, J. Geophys. Res., 113 A03S08, doi:10.1029/2007JA012558, 2008.

Friedel, R. H. W., Reeves, G. D., and Obara, T.: Relativistic electron dynamics in the inner magnetosphere-A review, J. Atmos. Sol. Terr. Phys., 64, 265, doi:10.1016/S1364-6826(01)00088-8, 2002.

Gallagher, D. L., Craven, P. D., and Comfort, R. H.: Global core plasma model, J. Geophys. Res., 105, 18819-18833, doi:10.1029/1999JA000241, 2000.

Glauert, S. A. and Horne, R. B.: Calculation of pitch angle and energy diffusion coefficients with the PADIE code, J. Geophys. Res., 110, A04206, doi:10.1029/2004JA010851, 2005.

Gulyaeva, T. L., Xueqin, H., Reinisch, B. W., and Bodo, W.: Plasmaspheric extension of topside electron density profiles, Adv. Space Res., 29, 825-831, 2002.

Gurnett, D. A. and Shaw, R. R.: Electromagnetic radiation trapped in the magnetosphere above the plasma frequency, J. Geophys. Res., 78, 8136-8149, doi:10.1029/JA078i034p08136, 1973.

Horne, R. B.: The contribution of wave particle interactions to electron loss and acceleration in the Earth's radiation belts during geomagnetic storms, in: Review of Radio Science 1999-2002, edited by: Stone, W. R., 801-828, chap. 33, 2002.

Horne, R. B., Glauert, S. A., and Thorne, R. M.: Resonant diffusion of radiation belt electrons by whistler-mode chorus, Geophys. Res. Lett., 30, 1493, doi:10.1029/2003GL016963, 2003.

Horne, R. B., Thorne, R. M., Glauert, S. A., Albert, J. M., Meredith, N. P., and Anderson, R. R.: Timescale for radiation belt electron acceleration by whistler mode chorus waves, J. Geophys. Res., 110, A03225, doi:10.1029/2004JA010811, 2005.

Horne, R. B., Thorne, R. M., Glauert, S. A., Meredith, N. P., Pokhotelov, D., and Santolík, O.: Electron acceleration in the Van Allen radiation belts by fast magnetosonic waves, Geophys. Res. Lett., 34, L17107, doi:10.1029/2007GL030267, 2007.

Horne, R. B., Kersten, T., Glauert, S. A., Meredith, N. P., Boscher, D., Sicard-Piet, A., Thorne, R. M., and Li, W.: A new diffusion matrix for whistler mode chorus waves, J. Geophys. Res. Space Phys., 118, 6302-6318, doi:10.1002/jgra.50594, 2013.
Jordanova, V. K., Albert, J., and Miyoshi, Y.: Relativistic electron precipitation by EMIC waves from self-consistent global simulations, J. Geophys. Res., 113, A00A10, doi:10.1029/2008JA013239, 2008.

Kennel, C. F. and Petschek, H. E.: Limit on stably trapped particle fluxes, J. Geophys. Res., 71, 1-28, doi:10.1029/JZ071i001p00001, 1966.

Kennel, C. F. and Engelmann, F.: Velocity Space Diffusion from Weak Plasma Turbulence in a Magnetic Field, Phys. Fluids, 9, 2377-2388, doi:10.1063/1.1761629, 1966.

Kim, H.-J. and Chan, A. A.: Fully adiabatic changes in storm-time relativistic electron fluxes, J. Geophys. Res., 102, 22107-22116, doi:10.1029/97JA01814, 1997.

Koons, H. C. and Roeder, J. L.: A survey of equatorial magnetospheric wave activity between 5 and 8 RE, Planet. Space Sci., 38, 1335-1341, doi:10.1016/0032-0633(90)90136-E, 1990.

Li, X. and Temerin, M.: The Electron Radiation Belt, Space Sci. Rev., 951, p. 569, 2001.

Li, W., Thorne, R. M., Meredith, N. P., Horne, R. B., Bortnik, J., Shprits, Y. Y., and Ni, B.: Evaluation of whistler mode chorus amplification during an injection event observed on CRRES, J. Geophys. Res., 113, A09210, doi:10.1029/2008JA013129, 2008.

Li, W., Thorne, R. M., Angelopoulos, V., Bonnell, J. W., McFadden, J. P., Carlson, C. W., LeContel, O., Roux, A., Glassmeier, K. H., and Auster, H. U.: Evaluation of whistler-mode chorus intensification on the nightside during an injection event observed on the THEMIS spacecraft, J. Geophys. Res., 114, A00C14, doi:10.1029/2008JA013554, 2009a.

Li, W., Thorne, R. M., Angelopoulos, V., Bortnik, J., Cully, C. M., Ni, B., LeContel, O., Roux, A., Auster, U., and Magnes, W.: Global distribution of whistler-mode chorus waves observed on the THEMIS spacecraft, Geophys. Res. Lett., 36, L09104, doi:10.1029/2009GL037595, 2009b.

Lyons, L. R.: General relations for resonant particle diffusion in pitch angle and energy, J. Plasma Phys., 12, 45-49, doi:10.1017/S0022377800024910, 1974a.

Lyons, L. R.: Pitch angle and energy diffusion coefficients from resonant interactions with ion-cyclotron and whistler waves, J. Plasma Phys., 12, 417, doi:10.1017/S002237780002537X, 1974b.

Lyons, L. R. and Thorne, R. M.: Equilibrium structure of radiation belt electrons, J. Geophys. Res., 78, 2142-2149, doi:10.1029/JA078i013p02142, 1973.

Lyons, L. R., Thorne, R. M., and Kennel, C. F.: Pitch-angle diffusion of radiation belt electrons within the plasmasphere, J. Geophys. Res., 77, 3455-3474, doi:10.1029/JA077i019p03455, 1972.

Meredith, N. P., Horne, R. B., Iles, R. H. A., Thorne, R. M., Anderson, R. R., and Heynderickx, D.: Outer zone relativistic electron acceleration associated with substorm-enhanced whistler mode chorus, J. Geophys. Res., 107, doi:10.1029/2001JA900146, 2002a.

Meredith, N. P., Horne, R. B., Summers, D., Thorne, R. M., Iles, R. H. A., Heynderickx, D., and Anderson, R. R.: Evidence for acceleration of outer zone electrons to relativistic energies by whistler mode chorus, Ann. Geophys., 20, 967-979, doi:10.5194/angeo20-967-2002, 2002b.

Meredith, N. P., Horne, R. B., Thorne, R. M., and Anderson, R. R.: Favored regions for chorus-driven electron acceleration to rela- 
tivistic energies in the Earth's outer radiation belt, Geophys. Res. Lett., 30, 1871, doi:10.1029/2003GL017698, 2003.

Meredith, N. P., Horne, R. B., Thorne, R. M., Summers, D., and Anderson, R. R.: Substorm dependence of plasmaspheric hiss, J. Geophys. Res., 109, A06209, doi:10.1029/2004JA010387, 2004.

Meredith, N. P., Horne, R. B., Glauert, S. A., Thorne, R. M., Summers, D., Albert, J. M., and Anderson, R. R.: Energetic outer zone electron loss timescales during low geomagnetic activity, J. Geophys. Res., 111, A05212, doi:10.1029/2005JA011206, 2006.

Meredith, N. P., Horne, R. B., Glauert, S. A., and Anderson, R. R.: Slot region electron loss timescales due to plasmaspheric hiss and lightning-generated whistlers, J. Geophys. Res., 112, A08214, doi:10.1029/2007JA012413, 2007.

Meredith, N. P., Horne, R. B., and Anderson, R. R.: Survey of magnetosonic waves and proton ring distributions in the Earth's inner magnetosphere, J. Geophys. Res., 113, A06213, doi:10.1029/2007JA012975, 2008.

Meredith, N. P., Horne, R. B., Glauert, S. A., Baker, D. N., Kanekal, S. G., and Albert, J. M.: Relativistic electron loss timescales in the slot region, J. Geophys. Res., 114, A03222, doi:10.1029/2008JA013889, 2009.

Meredith, N. P., Horne, R. B., Sicard-Piet, A., Boscher, D., Yearby, K. H., Li, W., and Thorne, R. M.: Global model of lower band and upper band chorus from multiple satellite observations, J. Geophys. Res., 117, A10225, doi:10.1029/2012JA017978, 2012.

Meredith, N. P., Horne, R. B., Bortnik, J., Thorne, R. M., Chen, L., Li, W., and Sicard-Piet, A.: Global statistical evidence for chorus as the embryonic source of plasmaspheric hiss, Geophys. Res. Lett., 40, 2891-2896, doi:10.1002/grl.50593, 2013.

Mourenas, D., Artemyev, A. V., Agapitov, O. V., and Krasnoselskikh, V.: Analytical estimates of electron quasi-linear diffusion by fast magnetosonic waves, J. Geophys. Res. Space Phys., 118, 3096-3112, doi:10.1002/jgra.50349, 2013.

Ni, B., Thorne, R. M., and Ma, Q.: Bounce-averaged Fokker-Planck diffusion equation in non-dipolar magnetic fields with applications to the Dungey magnetosphere, Ann. Geophys., 30, 733 750, doi:10.5194/angeo-30-733-2012, 2012.

Orlova, K. G. and Shprits, Y.: Dependence of pitch-angle scattering rates and loss timescales on the magnetic field model, Geophys. Res. Lett., 37, L05105, doi:10.1029/2009GL041639, 2010.

Ozhogin, P., Tu, J., Song, P., and Reinisch, B. W.: Field-aligned distribution of the plasmaspheric electron density: An empirical model derived from the IMAGE RPI measurements, J. Geophys. Res., 117, A06225, doi:10.1029/2011JA017330, 2012.

Santolík, O., Gurnett, D. A., Pickett, J. S., Parrot, M., and Cornilleau-Wehrlin, N.: A microscopic and nanoscopic view of storm-time chorus on 31 March 2001, Geophys. Res. Lett., 31, L02801, doi:10.1029/2003GL018757, 2004.

Santolik, O., Gurnett, D. A., Pickett, J. S., Parrot, M., and Cornilleau-Wehrlin, N.: Central Position of the Source Region of Storm-Time Chorus, Planet. Space Sci., 53, 299-305, 2005.
Sheeley, B. W., Moldwin, M. B., Rassoul, H. K., and Anderson, R. R.: An empirical plasmasphere and trough density model: CRRES observations, J. Geophys. Res., 106, 25631-25641, doi:10.1029/2000JA000286, 2001.

Sicard-Piet, A., Boscher, D., Bourdarie, S., Maget, V., and Lazaro, D.: Modélisation de l'environnement radiatif spatial, Technical report, RF 2/18475 DESP, 2011.

Summers, D. and Thorne, R. M.: Relativistic electron pitchangle scattering by electromagnetic ion cyclotron waves during geomagnetic storms, J. Geophys. Res., 108, 1143, doi:10.1029/2002JA009489, 2003.

Summers, D., Ni, B., and Meredith, N. P.: Timescales for radiation belt electron acceleration and loss due to resonant wave particle interactions: 2. Evaluation for VLF chorus, ELF hiss, and EMIC waves, J. Geophys. Res., 112, A04207, doi:10.1029/2006JA011993, 2007.

Thorne, R. M.: Radiation belt dynamics: The importance of wave-particle interactions, Geophys. Res. Lett., 37, L22107, doi:10.1029/2010GL044990, 2010.

Thorne, R. M., Horne, R. B., Glauert, S. A., Meredith, N. P., Shprits, Y. Y., Summers, D., and Anderson, R. R.: The influence of wave-particle interactions on relativistic electrons during storms, in Inner Magnetosphere Interactions: New Perspectives From Imaging, Geophys. Monogr. Ser., vol. 159, edited by: Burch, J., Schulz, M., and Spence, H., AGU, Washington DC, 2005.

Thorne, R. M., Li, W., Ni, B., Ma, Q., Bortnik, J., Chen, L., Baker, D. N., Spence, H. E., Reeves, G. D., Henderson, M. G., Kletzing, C. A., Kurth, W. S., Hospodarsky, G. B., Blake, J. B., Fennell, J. F., Claudepierre, S. G., and Kanekal, S. G.: Rapid local acceleration of relativistic radiation belt electrons by magnetospheric chorus, Nature, 504, 411-414, doi:10.1038/nature12889, 2013.

Tsurutani, B. T. and Smith, E. J.: Postmidnight chorus: A substorm phenomenon, J. Geophys. Res., 79, 118-127, doi:10.1029/JA079i001p00118, 1974.

Tsurutani, B. T. and Smith, E. J.: Two types of magnetospheric ELF chorus and their substorm dependences, J. Geophys. Res., 82, 5112-5128, doi:10.1029/JA082i032p05112, 1977.

Varotsou, A., Boscher, D., Bourdarie, S., Horne, R. B., Glauert, S. A., and Meredith, N. P.: Simulation of the outer radiation belt electrons near geosynchronous orbit including both radial diffusion and resonant interaction with Whistler-mode chorus waves, Geophys. Res. Lett., 32, L19106, doi:10.1029/2005GL023282, 2005.

Varotsou, A., Boscher, D., Bourdarie, S., Horne, R. B., Meredith, N. P., Glauert, S. A., and Friedel, R. H.: Three-dimensional test simulations of the outer radiation belt electron dynamics including electron-chorus resonant interactions, J. Geophys. Res., 113, A12212, doi:10.1029/2007JA012862, 2008. 